\title{
Bone radionuclide therapy and increased survival with radium-223 is the way to go for nuclear medicine: the offer that oncologists cannot refuse
}

\author{
Roberto C. Delgado Bolton ${ }^{1}$ (D) - Francesco Giammarile ${ }^{2}$
}

Received: 6 December 2017 / Accepted: 10 December 2017 / Published online: 14 January 2018

(C) Springer-Verlag GmbH Germany, part of Springer Nature 2018

In July 2013 The New England Journal of Medicine published a randomised controlled study demonstrating a survival benefit for castration-resistant prostate cancer (CRPC) patients with symptomatic bone metastases and no known visceral metastatic disease treated with radium-223 [1]. This study contributed to a change in paradigm in the treatment of CRPC patients, in whom until then radionuclide therapy had only a minor role. Nowadays, radium-223 is considered as one of the options in CRPC together with chemotherapy and radiotherapy. The breakthrough of radium-223 into the clinical arena is an example of how the Nuclear Medicine community should address the issue of getting to be considered a key player in oncology: providing high quality evidence and, if possible, demonstrating a survival benefit of our diagnostic and therapeutic procedures.

In the past 30 years Nuclear Medicine has had to fight its way into clinical indications and guidelines. The best example is ${ }^{18} \mathrm{~F}$-FDG PET, which after so many years is still struggling to be considered the standard diagnostic imaging test in some oncological indications where, based on expert opinion, it is clearly useful $[2,3]$. However, due to the lack of solid evidence, it is difficult, if not impossible, to appropriately discuss its inclusion in clinical algorithms. On one hand, this is quite a common situation, as obtaining high-quality evidence requires randomised controlled trials or very well designed and performed studies, that are very difficult to achieve in the clinical

Roberto C. Delgado Bolton rbiolton@gmail.com

1 Department of Diagnostic Imaging and Nuclear Medicine, University Hospital San Pedro and Centre for Biomedical Research of La Rioja (CIBIR), Logroño, La Rioja, Spain

2 International Atomic Energy Agency (IAEA), Nuclear Medicine and Diagnostic Imaging Section, Vienna, Austria field, especially when the preferred diagnostic test in guidelines is CT or MR [3]. When these are lacking, there is the possibility of performing systematic reviews and meta-analyses, which have become a way of providing evidence in difficult clinical questions [4-6]. On the other hand, we should be aware that we are competing with CT and MR, and while we are asked to provide high-quality evidence, in most instances CT and MR have been routinely used and included in clinical guidelines without having to provide the same evidence, mainly because, at the time they were introduced, there was nothing else. A clear example of the superiority of ${ }^{18} \mathrm{~F}-\mathrm{FDG}$ PET compared to CT is in the management of lymphomas [7].

Regarding theragnosis and radionuclide therapy, their potential is enormous. Both are becoming key players in several oncologic indications, as more and more evidence is available. Neuroendocrine neoplasms are an example of the potential of theragnosis, with PET tracers such as ${ }^{68}$ Ga-DOTA-conjugated somatostatin receptor targeting peptides to precisely diagnose the disease and ${ }^{177} \mathrm{Lu}$-DOTA peptides to accurately treat the disease [8]. However, the process is relatively slow given the multiple steps that are followed in the development and introduction of new radiopharmaceuticals. One limiting issue is the current legislation for new radiopharmaceuticals and radiation protection, which is being addressed in order to improve the translation from molecule to man [9]. Focusing on radionuclide therapy of bone metastases using radiopharmaceuticals labelled with beta emitting radionuclides such as strontium89 , samarium-153, and phosphorus-32, they present substantial advantages compared to other therapeutic options. Some of these advantages include their ability to simultaneously treat multiple sites of disease, easy administration, repeatability, and the possibility to integrate with other treatments.

In this issue two EANM guidelines focusing on radionuclide therapy are presented, one on the treatment with radium223 of metastatic castration-resistant prostate cancer [10] and, 
the other, dedicated to therapy of bone metastases with radiopharmaceuticals labelled with beta emitting radionuclides [11]. The methodology applied in both guidelines has included a systematic literature search with PICO questionnaires, and the application of SIGN checklists and SIGN grading for the evaluation of the level of evidence. The aim of both guidelines is to assist Nuclear Medicine specialists when evaluating patients who might be candidates for these treatments, and help applying these treatments and managing the procedures and follow-up of the patients.

\section{Compliance with ethical standards}

Conflict of interest The authors declare that they have no conflict of interest.

Ethical approval This article does not contain any studies with human participants or animals performed by any of the authors. This is not a research article.

Informed consent No informed consent was needed.

\section{References}

1. Parker C, Nilsson S, Heinrich D, Helle SI, O’Sullivan JM, Fossa $\mathrm{SD}$, et al. Alpha emitter radium-223 and survival in metastatic prostate cancer. N Engl J Med. 2013;369:213-23.

2. Boellaard R, Delgado-Bolton R, Oyen WJ, Giammarile F, Tatsch $\mathrm{K}$, Eschner W, et al. FDG PET/CT: EANM procedure guidelines for tumour imaging: version 2.0. Eur J Nucl Med Mol Imaging. 2015;42:328-54.

3. Jadvar H, Colletti P, Delgado-Bolton R, Esposito G, Krause BJ, Iagaru $\mathrm{AH}$, et al. Appropriate use criteria for FDG PET/CT restaging and response assessment of malignant disease. $\mathrm{J}$ Nucl Med. 2017;58:2026-37.

4. Delgado-Bolton RC, Fernández-Pérez C, González-Maté A, Carreras JL. Meta-analysis of the performance of ${ }^{18}$ F-FDG PET in primary tumor detection in unknown primary tumors. $\mathrm{J}$ Nucl Med. 2003;44:1301-14.

5. Jiménez-Requena F, Delgado-Bolton RC, Fernández-Pérez C, Gambhir SS, Schwimmer J, Pérez-Vázquez JM, et al. Metaanalysis of the performance of (18)F-FDG PET in cutaneous melanoma. Eur J Nucl Med Mol Imaging. 2010;37:284-300.

6. García Vicente AM, Delgado-Bolton RC, Amo-Salas M, LópezFidalgo J, Caresia Aróztegui AP, García Garzón JR, et al. 18Ffluorodeoxyglucose positron emission tomography in the diagnosis of malignancy in patients with paraneoplastic neurological syndrome: a systematic review and metaanalysis. Eur J Nucl Med Mol Imaging. 2017;44:1575-87.

7. Goméz León N, Delgado-Bolton RC, del Campo del Val L, Cabezas B, Arranz R, García M, et al. Multicenter comparison of contrast-enhanced FDG PET/CT and 64-slice multi-detector CT for initial staging and response evaluation at the end of treatment in patients with lymphoma. Clin Nucl Med. 2017;42:595-602.

8. Bozkurt MF, Virgolini I, Bagolova S, Beheshti M, Rubello D, Decristoforo $\mathrm{C}$, et al. Guideline for PET/CT imaging in neuroendocrine neoplasms with ${ }^{68} \mathrm{Ga}$-DOTA-conjugated somatostatin receptor targeting peptides and ${ }^{18} \mathrm{~F}-\mathrm{DOPA}$. Eur J Nucl Med Mol Imaging. 2017;44:1588-601.

9. Giammarile F, Muylle K, Delgado Bolton R, Kunikowska J, Haberkorn U, Oyen W. Dosimetry in clinical radionuclide therapy: the devil is in the detail. Eur J Nucl Med Mol Imaging. 2017;44:13.

10. Poeppel TD, Handkiewicz-Junak D, Andreeff M, Becherer A, Bockisch A, Fricke E, et al. EANM Guideline for radionuclide therapy with radium-223 of metastatic castration-resistant prostate cancer. Eur J Nucl Med Mol Imaging. 2017 Dec 12. https://doi.org/ 10.1007/s00259-017-3900-4.

11. Handkiewicz-Junak D, Poeppel TD, Bodei L, Aktolum C, Ezziddin $\mathrm{S}$, Giammarile F, et al. EANM Guideline for radionuclide therapy of bone metastases. Eur J Nucl Med Mol Imaging. 2017. https://doi. org/10.1007/s00259-018-3947-x. 